\title{
JNS Review Recognition 2019
}

Journal of Nutritional Science (2019), vol. 8, e37, page 1 of 1

doi:10.1017/jns.2019.35

The Editorial Board of the Journal of Nutritional Science would like to thank the following for their contribution as peer reviewers in 2019:

Folasade Adebayo

Benjamin Allès

Naser Alsharairi

Rona Antoni

Bernardo Baldisserotto

Marc Bomhof

Andrew Bottley

Maria Bouga

Roger Bouillon

Kathryn Bradbury

Alex Brito

Danyel Bueno-Dalto

Daniela Canella

Monica Carlsen

Lucy Cheke

Raphaël Chouinard-Watkins

Miriam Clegg

Adam Collins

Karen Corbin

Diana Coulon

Marta Crous Bou

Willem De Keyzer

Tim De Meyer

Saskia de Pee

Paraskeui Detopoulou

Georgina Dodd

Lauren Duckworth

Alastair Duncan

Peter Eckl

Samer El-Kadi

Aya Fujiwara
Melawhy Garcia

Yong Meng Goh

Horst Göring

Sueppong Gowachirapant

Peter Grabowski

Neil Harris

Anne Hatloy

Stephen Hennigar

Adela Hruby

Hiroshi Ichikawa

Roberta Imperatore

Arora Ingadottir

Suvi Itkonen

Sandra Iuliano

Hélène Jacques

Xia Jiang

Cyril Kendall

Svyatoslav Komarnytsky

Meron Lewis

Dongmin Liu

Trias Mahmudiono

Emma McMahon

Anine Medin

Jelena Meinila

J. Bernadette Moore

Alyssa Moran

Charlotte Mortensen

Laurent Mosoni

Peter Mount

Anarina Murillo

David Neiman
Tzortzis Nomikos

Signe Okkels

Chad Paton

Jaqueline Pereira

Julia Peterson

Krista Power

Damar Pramusinto

Anabelle Retondario

Caroline Richard

Christian Ritz

Asman Sadino

Rodrigo San-Cristobal

Jonathan Scott

Cecilia Severi

Harbindar Singh

Kylie Smith

Daniel So

Michelle Swainson

Carmen Tekwe

Adriana van Ballegooijen

Corné van Dooren

María Victoria Velarde

Joseph Wakshlag

Linlin Wang

Lynn Weber

Jean Welsh

Emma Wightman

Hiroshi Yokomichi

Satoko Yoneyama

(C) The Author(s) 2019. This is an Open Access article, distributed under the terms of the Creative Commons Attribution licence (http://creative commons.org/licenses/by/4.0/), which permits unrestricted re-use, distribution, and reproduction in any medium, provided the original work is properly cited 